\title{
A simple chemical test to distinguish mycobacteria from other mycolic-acid-containing actinomycetes
}

\author{
Mohamed E. Hamid, ${ }^{1,2}$ David E. Minnikin ${ }^{1}$ and Michafl Goodfellow ${ }^{2 *}$ \\ Departments of ${ }^{1}$ Chemistry and ${ }^{2}$ Microbiology, University of Newcastle, Newcastle upon Tyne NEI $7 R U, U K$
}

(Received 25 January 1993; revised 27 March 1993; accepted 13 April 1993)

\begin{abstract}
Two hundred and fifty-two representatives of the genera Corynebacterium, Gordona, Mycobacterium, Nocardia, Rhodococcus and Tsukamurella were degraded by alkaline hydrolysis and their mycolic acids extracted as methyl esters following phase-transfer-catalysed esterification. When the mycolic acid methyl esters were treated with a mixture of acetonitrile and toluene all mycobacterial mycolates formed copious white precipitates whereas all but 5 out of the 106 non-mycobacterial mycolates remained in solution. The precipitated methyl mycolates and the dried soluble mycolates were compared by pyrolysis gas chromatography and silica gel thin-layer chromatography. On pyrolysis, the precipitated methyl mycolates from mycobacteria yielded fatty acid methyl esters with 20 to 26 carbon atoms whereas those from the remaining taxa produced shorter-chain esters. Mycobacteria and Tsukamurella paurometabola gave multispot mycolic acid patterns on thin-layer chromatography of their methyl esters whereas those from the remaining strains gave single spots. Our results indicate that Rhodococcus chlorophenolicus strains contain mycolic acids typical of mycobacteria. It can be concluded that the mycolic acid precipitation test provides a simple and reliable way of distinguishing mycobacteria from all other prokaryotes, notably from other mycolic-acid-containing taxa.
\end{abstract}

\section{Introduction}

Actinomycetes containing mycolic acids (long-chain 2alkyl 3-hydroxy fatty acids) have many properties in common (Bowden \& Goodfellow, 1990; Goodfellow, 1991) and form a distinct suprageneric taxon that encompasses the genera Corynebacterium, Gordona, Mycobacterium, Nocardia, Rhodococcus and Tsukamurella (Collins et al., 1988; Stackebrandt et al., 1988). Members of these taxa are currently distinguished using a combination of chemical and morphological markers (Bowden \& Goodfellow, 1990; Goodfellow, 1991) but improved diagnostic tests are needed, particularly for separating mycobacteria of clinical and veterinary significance from all other mycolic-acid-containing organisms.

Distinguishing mycobacteria from other mycolic-acidcontaining taxa presently depends upon the application of chemotaxonomic techniques designed to separate different mycolic acid types in whole-organism methanolysates (Goodfellow, 1991). Mycolic acids vary considerably in structure, ranging from the relatively simple

*Author for correspondence. Tel. (091) 222 7706; fax (091) 261 1182 .

Abbreviation: MAME, mycolic acid methyl ester(s). mixtures of saturated and unsaturated acids found in corynebacteria to the very complicated mixtures characteristic of mycobacteria (Minnikin et al., 1975, 1984a,b, 1985). Several simple chemical procedures are available to distinguish between the various types of mycolic acids but none of them unambiguously separates all mycobacteria from the remaining mycolic-acid-containing taxa.

Qualitative evaluation of mycolic acids can be achieved using TLC (Minnikin et al., 1975): methanolysates of mycobacteria give multispot mycolate patterns whereas those of corynebacteria, gordonae, nocardiae and rhodococci give single spots whose mobilities reflect the chain length and structure of the constituent mycolic acids. The patterns of mycolic acid types can also be used for the identification of mycobacterial species (Minnikin et al., 1984a,b, 1985). Mycobacterium fallax and Mycobacterium triviale strains, however, give single mycolic acid spots on TLC analysis (Dobson et al., 1985) and can thereby be confused with gordonae and nocardiae. Moreover, the multispot mycolic acid pattern characteristic of most mycobacteria is also shown by some Tsukamurella paurometabola strains (Goodfellow et al., 1978; Collins et al., 1988); the mycolates from strains of this species separate into two components according to the presence or absence of a double bond at the 2-

$0001-8110 \bigodot 1993 \mathrm{SGM}$ 
Table 1. Designation, source and history of test strains

\begin{tabular}{|c|c|}
\hline $\begin{array}{l}\text { Designation and } \\
\text { laboratory number }\end{array}$ & Source and strain history \\
\hline \multicolumn{2}{|l|}{$\begin{array}{l}\text { Genus Corynebacterium: } \\
\text { C. bovis }\end{array}$} \\
\hline${ }^{*} \mathrm{C} 12$ & NCTC 3224 ; A. E. Evans, NIH Washington in 1930 ; milk \\
\hline $\begin{array}{l}\text { C. cystitidis } \\
{ }^{*} \text { C95 }\end{array}$ & \\
\hline $\begin{array}{l}{ }^{*} \mathrm{C} 95 \\
\text { C. diphtheriae }\end{array}$ & NCTC 11863; ATCC 29593; R. Yanagawa, 42; K. Hirato, cow \\
\hline $\mathrm{C} 97 \pm$ & $\begin{array}{l}\text { PW8, A. B. F. Standfast, The Lister Institute of Preventive } \\
\text { Medicine, Elstree, UK }\end{array}$ \\
\hline \multicolumn{2}{|l|}{ C. diphtheriae var. gravis } \\
\hline \multicolumn{2}{|r|}{ 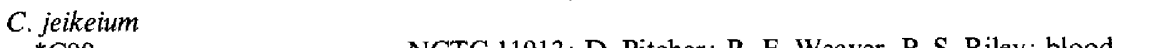 } \\
\hline${ }^{*} \mathrm{C} 98$ & NCTC 11913; D. Pitcher; R. E. Weaver, P. S. Riley; blood \\
\hline $\mathrm{C} 99$ & NCTC 11914; D. Pitcher; S. Eykyn; blood \\
\hline $\mathrm{C} 100, \mathrm{C} 101$ & $\begin{array}{l}\text { NCTC 11915, 11917; D. Pitcher; R. Harding, axilla and } \\
\text { vagina, respectively. }\end{array}$ \\
\hline \multicolumn{2}{|r|}{ 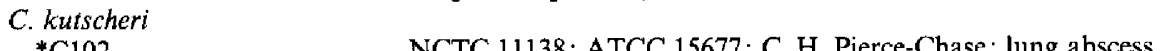 } \\
\hline${ }^{*} \mathrm{C} 102$ & $\begin{array}{l}\text { NCTC } 11138 ; \text { ATCC 15677; C. H. Pierce-Chase; lung abscess, } \\
\text { mouse }\end{array}$ \\
\hline \multicolumn{2}{|l|}{ C. matruchotii } \\
\hline \multicolumn{2}{|l|}{ C. minutissimum } \\
\hline \multicolumn{2}{|l|}{ C. mycetoides } \\
\hline *C63 & NCTC 9864; A. Castellani \\
\hline \multicolumn{2}{|l|}{ C. pilosum } \\
\hline${ }^{*} \mathrm{C} 105$ & $\begin{array}{l}\text { NCTC 11862; ATCC 29592; R. Yanagawa } 46 \text { Hara; } \\
\text { K. Hirato; cow }\end{array}$ \\
\hline C. pseudodiphtheriticum & NCTC 11136; ATCC 10700; J. M. Coffey, 153. \\
\hline $\begin{array}{l}\text { C. pseudotuberculosis } \\
{ }^{*} \mathrm{Cl} 5\end{array}$ & $\begin{array}{l}\text { NCTC } 3450 ; \text { G. F. Petrie; J. B. Buxton; infected gland of } \\
\text { sheep, South America }\end{array}$ \\
\hline \multicolumn{2}{|r|}{ NCTC 7448; B. Weitz, cow } \\
\hline \multicolumn{2}{|l|}{ C. striatum } \\
\hline C. variabilis & NCTC 764; F. C. Minnett \\
\hline${ }^{*} \mathrm{C} 357$ & $\begin{array}{l}\text { NCDO 2097; W. H. J. Crombach (Caseobacter polymorphus), } \\
\text { AC920; Meshanger cheese }\end{array}$ \\
\hline \multicolumn{2}{|l|}{$\begin{array}{l}\text { Genus Gordona: } \\
\text { G. bronchialis }\end{array}$} \\
\hline$*$ N654 & $\begin{array}{l}\text { NCTC } 10677 ; \text { M. Tsukamura, } 3410 ; \text { H. Kondo; sputum, } \\
\text { pulmonary lesion }\end{array}$ \\
\hline N1058 & $\begin{array}{l}\text { C. K. Campbell, London School of Hygiene and Tropical } \\
\text { Medicine, UK, 5424, breast abscess }\end{array}$ \\
\hline \multicolumn{2}{|l|}{ G. rubropertincta } \\
\hline *N4 & $\begin{array}{l}\text { NCIB 9664; ATCC 14352; R. S. Breed; R. E. Gordon, 154; } \\
\text { soil }\end{array}$ \\
\hline \multicolumn{2}{|r|}{ Son } \\
\hline N931 & $\begin{array}{l}\text { M. Tsukamura, The National Chubu Hospital, Obu, Aichi, } \\
\text { Japan, 6130; sputum }\end{array}$ \\
\hline \multicolumn{2}{|r|}{ 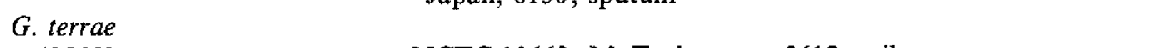 } \\
\hline *N659 & NCTC 10669; M. Tsukamura, 3612, soil \\
\hline N888 & M. Tsukamura, 3603 ; soil \\
\hline \multicolumn{2}{|l|}{$\begin{array}{l}\text { Genus Mycobacterium: } \\
\text { M. abscessus }\end{array}$} \\
\hline $\begin{array}{l}\text { M100 } \\
\text { M. aichiensis }\end{array}$ & NCTC 10882 ; C. A. Morris, KH 63330 ; cervical lymph node \\
\hline $\begin{array}{l}\text { M. aichiensis } \\
{ }^{*} \mathrm{M} 251\end{array}$ & NCTC 10820; M. Tsukamura, 49005; soil \\
\hline $\begin{array}{l}\text { M. aurum } \\
* \mathrm{M} 12\end{array}$ & NCTC 10437 ; M. Tsukamura, biotype 1, 358 \\
\hline M. avium & \\
\hline M504‡ & $\begin{array}{l}\text { H. B. Lee, Central Veterinary Laboratory, Weybridge, UK, } \\
\text { M. avium D4 }\end{array}$ \\
\hline
\end{tabular}


Table 1. (cont.)

\begin{tabular}{|c|c|}
\hline $\begin{array}{l}\text { Designation and } \\
\text { laboratory number }\end{array}$ & Source and strain history \\
\hline \multicolumn{2}{|l|}{ 'M. borstelense" } \\
\hline *M492 & M. Tsukamura, 19009; ATCC 19235 \\
\hline M488-M491 & $\begin{array}{l}\text { M. Tsukamura, 19003, 19004, 19005, 19006; R. N. Bönicke, } \\
\text { SN283, SN284, SN275, SN288 }\end{array}$ \\
\hline M547 & $\begin{array}{l}\text { U. Krallmann-Wenzel, Institute for Experimental Biology and } \\
\text { Medicine, Borstel, Germany, SN287 }\end{array}$ \\
\hline \multicolumn{2}{|r|}{ 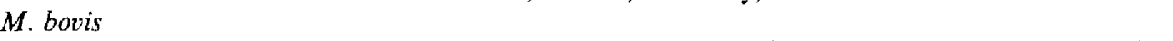 } \\
\hline M460t & $\begin{array}{l}\text { M. Magnusson, Statens Seruminstitut, Copenhagen, Denmark, } \\
\text { MNC } 27\end{array}$ \\
\hline \multicolumn{2}{|r|}{ - } \\
\hline M461 & NCTC 5692 ; A. S. Griffith; A. Calmette \\
\hline \multicolumn{2}{|l|}{ M. chelonei } \\
\hline *M350 & NCTC 946; F. M. Perry; tortoise tubercle \\
\hline M438 & $\begin{array}{l}\text { P. A. Jenkins, PHLS Mycobacterium Reference Unit, } \\
\text { University Hospital of Wales, Heath Park, Cardiff, UK, } \\
3330 \text {; abscess on arm }\end{array}$ \\
\hline M439 & P. A. Jenkins, 3622 ; ulcer on foot \\
\hline $\mathrm{M} 440, \mathrm{M} 442$ & P. A. Jenkins, 7883,13152 ; abscesses on hip \\
\hline M441 & P. A. Jenkins, 9920 ; pus - site unspecified \\
\hline M444 & P. A. Jenkins, 17714 ; M. Kubica \\
\hline M445 & P. A. Jenkins, 18014; abscess \\
\hline M446 & P. A. Jenkins, 19030; abscess on tibia \\
\hline M447 & P. A. Jenkins, 19410 ; lymph node \\
\hline \multicolumn{2}{|l|}{$M$. chitae } \\
\hline$* \mathrm{M} 101$ & NCTC 10485; M. Tsukamura, 2502; soil \\
\hline \multicolumn{2}{|r|}{ (2) } \\
\hline *M165 & NCTC 358 \\
\hline \multicolumn{2}{|l|}{ M. fallax } \\
\hline$* \mathrm{M} 432$ & $\begin{array}{l}\text { V. Lévy-Frébault, Institut Pasteur, Paris, France, CIPT } 1390003 \\
\text { (CIP 8139), clinical strain }\end{array}$ \\
\hline M428-M431 & V. Lévy-Frébault, 54, 80, 81, 206; River Seine \\
\hline \multicolumn{2}{|l|}{ M. farcinogenes } \\
\hline${ }^{*} \mathrm{M} 262$ & $\begin{array}{l}\text { NCTC 10955; P. Perreau; G. Chamoiseau, IEMVT } 75 \text {, bovine } \\
\text { farcy, lymph node }\end{array}$ \\
\hline M260, M261 & $\begin{array}{l}\text { M. P. Lechevalier, Rutgers University, New Brunswick, USA: } \\
\text { Institut Pasteur (IP), Paris, 736, 740; bovine farcy, Farcha } \\
\text { Laboratory, Fort Lamy, Chad }\end{array}$ \\
\hline $\mathrm{M} 269, \mathrm{M} 270$ & $\begin{array}{l}\text { E. H. Runyon, Tuberculosis Research Institute, Pretoria, South } \\
\text { Africa, R422, R423; IP735, IP736; bovine farcy, Farcha } \\
\text { Laboratory, Fort Lamy, Chad }\end{array}$ \\
\hline M271 & $\begin{array}{l}\text { E. H. Runyon, R415; R. E. Gordon, 1226; bovine farcy, } \\
\text { Farcha Laboratory, Fort Lamy, Chad }\end{array}$ \\
\hline $\mathrm{M} 272-\mathrm{M} 277$ & $\begin{array}{l}\text { E. H. Runyon, R425, R414, R426, R427, R428, R429; IP738, } \\
\text { IP739, IP740, IP742, IP743, IP744; bovine farcy, Farcha } \\
\text { Laboratory, Fort Lamy, Chad }\end{array}$ \\
\hline M278 & $\begin{array}{l}\text { E. H. Runyon, R430; R. E. Gordon, 1224; IP745; bovine } \\
\text { farcy, Farcha Laboratory, Fort Lamy, Chad }\end{array}$ \\
\hline $\mathrm{M} 280, \mathrm{M} 281$ & E. H. Runyon, R147, R457; P. Perreau, MA75, MA938 \\
\hline $\mathrm{M} 284, \mathrm{M} 285$ & $\begin{array}{l}\text { E. H. Runyon, R416, 417; R. E. Gordon, 1244, 1243; J. E. } \\
\text { Mostafa, Khartoum, Sudan }\end{array}$ \\
\hline M462-M467 & $\begin{array}{l}\text { M. Abdulle, Serum and Vaccine Institute, P.O. Box } 919, \\
\text { Mogadishu, MA5, MA9, MA30, MA39, MA191, MA192, } \\
\text { Somalia; bovine farcy, skin lesions, Somalia }\end{array}$ \\
\hline M468-M470 & $\begin{array}{l}\text { M. Abdulle, MA217, MA612, MA768, bovine farcy, lymph } \\
\text { nodes, Somalia }\end{array}$ \\
\hline M471 & M. Abdulle, MA555, bovine farcy, mammary gland, Somalia \\
\hline M472 & M. Abdulle, MA687, bovine farcy, liver, Somalia \\
\hline M473 & M. Abdulle, MA785, bovine farcy, lung, Somalia \\
\hline M474 & M. E. Hamid, MH15, bovine farcy, skin biopsy, Sudan \\
\hline M475-M477 & $\begin{array}{l}\text { M. E. Hamid, MH16, MH62, MH67, bovine farcy, lymph } \\
\text { nodes, Sudan }\end{array}$ \\
\hline M478-M485 & $\begin{array}{l}\text { S. M. El-Sanousi, Department of Microbiology, Faculty of } \\
\text { Veterinary Science, PO Box 32, Khartoum North, Sudan; } \\
\text { MS52 (KHF9), MS54 (KHF1), MS55 (KF8), MS57 (KF6), } \\
\text { MS59 (KF16), MS60 (NF6), MS63 (NF10), MS64 (NF12) } \\
\text { bovine farcy, lymph nodes, Sudan }\end{array}$ \\
\hline
\end{tabular}


Table 1. (cont.)

\begin{tabular}{|c|c|}
\hline $\begin{array}{l}\text { Designation and } \\
\text { laboratory number }\end{array}$ & Source and strain history \\
\hline N710†, M711 & $\begin{array}{l}\text { M. Ridell, Institute of Medical Microbiology, University of } \\
\text { Gothenburg, Sweden, GB041, GB042; R. E. Gordon, 1226, } \\
\text { 1242; bovine farcy, Farcha Laboratory, Fort Lamy, Chad }\end{array}$ \\
\hline N715†, N719, N720 & $\begin{array}{l}\text { M. Ridell, GB046, GB051, GB052; M. P. Lechevalier, 1224, } \\
\text { 1225, 1244; bovine farcy, Farcha Laboratory, Fort Lamy, } \\
\text { Chad }\end{array}$ \\
\hline $\mathrm{N} 725 \dagger$ & $\begin{array}{l}\text { M. Ridell, GA } 833 \text {; M. Magnusson, } 749 \text {; bovine farcy, Farcha } \\
\text { Laboratory, Fort Lamy, Chad }\end{array}$ \\
\hline \multicolumn{2}{|l|}{ M. fortuitum } \\
\hline *M204 & NCTC 10394; R. E. Gordon; J. C. Cruz 1; cold abscess \\
\hline M205 & NCTC $10395 ;$ R. E. Gordon; J. C. Cruz 2; cold abscess \\
\hline M368 & M. Ridell, GA023; Institute Superiore di Sanita, Rome, 456 \\
\hline M369† & M. Ridell, GB178; ATCC 6841 \\
\hline M390 & $\begin{array}{l}\text { NCTC } 2291 ; \text { A. Stanley Griffith, roe of halibut (Hippoglossus } \\
\text { vulgaris) }\end{array}$ \\
\hline $\begin{array}{l}\text { M506-M512, M514, M516, } \\
\text { M522, M528, M531, M534, } \\
\text { M538, M539-M542 }\end{array}$ & $\begin{array}{l}\text { J. M. Grange, School of Pathology, Middlesex Hospital, } \\
\text { London, UK, } 6,9,11,13,14,15,17,21,29,47,58,67,79 \text {, } \\
86,93,94,98,101\end{array}$ \\
\hline \\
\hline${ }^{*} \mathrm{M} 495$ & NCTC 10267; L. F. Bojalil; E. H. Runyon; gastric lavage \\
\hline $\begin{array}{l}\text { M. kansasii } \\
\text { M496 }\end{array}$ & NCTC 10268 ; L. F. Bojalil; E. H. Runyon \\
\hline \multicolumn{2}{|r|}{ 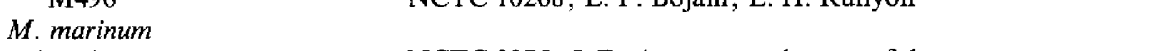 } \\
\hline $\begin{array}{l}{ }^{*} \mathrm{M} 181 \\
\text { M. microti }\end{array}$ & NCTC 2275; J. D. Aronson; salt-water fish \\
\hline $\begin{array}{l}\text { M. microti } \\
\quad * \mathrm{M} 497\end{array}$ & NCTC 8710; A. Q. Wells, L V 285/303; vole \\
\hline \multicolumn{2}{|l|}{ M. neoaurum } \\
\hline \multicolumn{2}{|r|}{ NCTC 10818; M. Tsukamura, 3503; sonl } \\
\hline \multicolumn{2}{|r|}{ NCTC 10424; M. Tsukamura, 317; soil } \\
\hline M. parafortuitum & NCIC 107/8; M. Isukamura, 4588; N. Kita; sputum \\
\hline \multicolumn{2}{|l|}{ M. peregrinum } \\
\hline *M6 & NCTC 10264 ; L. F. Bojalil, 6020 ; bronchial aspirate of child \\
\hline M418-M423, M427 & $\begin{array}{l}\text { P. A. Jenkins, } 7678,7680,14654,14657,14894,14895,19774 \text {; } \\
\text { sputum }\end{array}$ \\
\hline M424 & P. A. Jenkins, 16788 ; peritoneal fluid \\
\hline M425, M426 & P. A. Jenkins, $17564 ; 14462$; urine \\
\hline \multicolumn{2}{|l|}{ M. phlei } \\
\hline M206 & NCTC 10266; L. F. Bojalil; W. Steenken Jr \\
\hline $\begin{array}{l}\text { M. rhodesiae } \\
{ }_{*} \mathrm{M} 176\end{array}$ & NCTC 10779; M. Tsukamura, 5295; L. King; sputum \\
\hline \multicolumn{2}{|r|}{ NCIC 107/9; M. I sukamura, 5295; L. King; sputum } \\
\hline${ }^{*} \mathrm{M} 500$ & $\begin{array}{l}\text { NCTC 10803; ATCC 19981; P. Hauduroy; F. H. Prissick; } \\
\text { cervical lymph node }\end{array}$ \\
\hline \multicolumn{2}{|l|}{$M$. senegalense } \\
\hline${ }^{*} \mathrm{M} 263$ & $\begin{array}{l}\text { NCTC 10956; P. Perreau; G. Chamoiseau, IEMVT 378; } \\
\text { bovine lymph node }\end{array}$ \\
\hline M259 & M. P. Lechevalier; M. Goodfellow, W34; NCTC 4524 \\
\hline M264, M265, M266 & $\begin{array}{l}\text { E. H. Runyon, R397, R408, R409, R410; R. E. Gordon, 1360, } \\
\text { 1361, 1362, M2681364; IP369, IP397, IP433C, IP425C; } \\
\text { bovine farcy, Dakar, Senegal }\end{array}$ \\
\hline M279, M282, M283 & $\begin{array}{l}\text { E. H. Runyon, R148, R455, R456; P. Perreau, MA378, D1, } \\
\text { D2 }\end{array}$ \\
\hline N714 & $\begin{array}{l}\text { M. Ridell, GB045; R. E. Gordon, R1363, bovine farcy, Dakar, } \\
\text { Senegal }\end{array}$ \\
\hline N717†, N718, N721 & $\begin{array}{l}\text { M. Ridell, GB048, GB049, GB053; M. P. Lechevalier, 1362, } \\
\text { 1364, 931; bovine farcy, Dakar, Senegal }\end{array}$ \\
\hline N723 & M. Ridell, GA816; R. N. Bönicke, SN 5701 \\
\hline N726, N728 & $\begin{array}{l}\text { M. Ridell, GA881, GA883; M. Magnusson, 874, 876; bovine } \\
\text { farcy, Dakar, Senegal }\end{array}$ \\
\hline \multicolumn{2}{|l|}{ M. smegmatis } \\
\hline${ }^{* \mathrm{M} 216}$ & $\begin{array}{l}\text { J. M. Grange, 2; NCTC } 8159 \text {; R. E. Gordon, Cornell } 3 \\
\text { NCTC } 8159 \text {; R. E. Gordon. Cornell } 3\end{array}$ \\
\hline
\end{tabular}


Table 1. (cont.)

\begin{tabular}{|c|c|}
\hline $\begin{array}{l}\text { Designation and } \\
\text { laboratory number }\end{array}$ & Source and strain history \\
\hline M211 & NCTC $10265 ;$ L. F. Bojalil; S. A. Waksman \\
\hline M217 & J. M. Grange, 77; NCTC 10265; L. F. Bojalil; S. A. Waksman \\
\hline M218 & J. M. Grange, 138; milk \\
\hline \multicolumn{2}{|l|}{${ }^{*} M$. triviale } \\
\hline M501 & ATCC 23292 \\
\hline \multicolumn{2}{|l|}{ M. tuberculosis } \\
\hline M502 & NCTC 7416; ATCC 9360, strain $\mathrm{H}_{37 R_{\mathrm{V}}}$ \\
\hline M503+ & $\begin{array}{l}\text { H. B. Lee, Central Veterinary Laboratory, Weybridge, UK, } \\
\text { M. tuberculosis C }\end{array}$ \\
\hline \multicolumn{2}{|r|}{ (1) } \\
\hline${ }^{*} \mathrm{M} 302$ & NCTC 10916; ATCC 15483; R. N. Bönicke, SN 920 \\
\hline \multicolumn{2}{|l|}{$\begin{array}{l}\text { Genus Nocardia } \\
\text { Nocardia amarae }\end{array}$} \\
\hline${ }^{*} \mathrm{~N} 667$ & $\begin{array}{l}\text { M. P. Lechevalier, Se } 6 \text {; foaming activated sludge, Andover } \\
\text { (Miami), Florida, USA }\end{array}$ \\
\hline N787 & M. Lechevalier, Se 291 ; Somerset-Raritan, NJ, USA \\
\hline \multicolumn{2}{|r|}{ 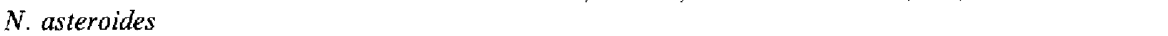 } \\
\hline *N317 & $\begin{array}{l}\text { ATCC } 19247 ; \text { R. E. Gordon, } 727 \text {; L. Ajello, M170-6; } \\
\text { W. Bowman }\end{array}$ \\
\hline N13 & $\begin{array}{l}\text { NCTC } 8595 \text {; strain R. H. Gorrill, Salonika, Greece, pus of } \\
\text { empyema }\end{array}$ \\
\hline N97 & R. E. Gordon, N659 \\
\hline N105 & $\begin{array}{l}\text { R. E. Gordon, } 9969 \text {; ATCC } 9969 \text {; C. W. Emmons, } 9955 \text {; } \\
\text { P. Hadley; generalized mycetoma }\end{array}$ \\
\hline $\mathrm{N} 201$ & CBS 51467 ; plasterwork \\
\hline $\mathrm{N} 216$ & $\begin{array}{l}\text { J. E. Thiemann, Lepetit, Milan, Italy, S547; PSA 161; IMRU } \\
652 \text {; E. N. Azarowicz, 110; A. Gonzáles-Ochoa, 1139; A. L. } \\
\text { Carrión }\end{array}$ \\
\hline N688-N692, N695 & $\begin{array}{l}\text { K. P. Schaal, Hygiene Institute, Cologne, Germany, N2, N3, } \\
\text { N5, N9, N23, N67 }\end{array}$ \\
\hline \multicolumn{2}{|r|}{ W } \\
\hline *N318 & $\begin{array}{l}\text { ATCC 19296; R. E. Gordon, IMRU 845; J. Schneidau, Jr 381; } \\
\text { A. Batista, 631; Institut Pasteur, Paris, } 337\end{array}$ \\
\hline \multicolumn{2}{|r|}{ 2. L } \\
\hline *N64I & J. Lacey, A 444; M. P. Lechevalier, $1086 \mathrm{~W}$ \\
\hline \multicolumn{2}{|l|}{ N. carnea } \\
\hline *N675 & $\begin{array}{l}\text { R. E. Gordon, 3419; ATCC 6847; NCTC } 3527 \text { (Actinomyces } \\
\text { carneus); V. Puntoni, } 30\end{array}$ \\
\hline \multicolumn{2}{|l|}{ N. farcinica } \\
\hline$*$ N699 & $\begin{array}{l}\text { ATCC } 3318 ; \text { S. A. Waksman, strain } 3318 \text { (Nocard's original } \\
\text { strain) }\end{array}$ \\
\hline $\mathrm{N} 258 \dagger$ & M. P. Lechevalier, ATCC 3318; S. A. Waksman's 3318 \\
\hline N668 & $\begin{array}{l}\text { S. G. Bradley, Virginia Commonwealth University, Richmond, } \\
\text { VA; Georgia, USA, VAC } 462\end{array}$ \\
\hline N669 & S. G. Bradley, VAC 300 \\
\hline N671† & $\begin{array}{l}\text { S. G. Bradley, VAC 330, ATCC } 3318 \text {; R. E. Gordon; S. A. } \\
\text { Waksman } 3318\end{array}$ \\
\hline N687 & K. P. Schaal, NI; strain Berlin \\
\hline N701 & M. Ridell, N67; M. Magnusson, 753; ATCC 3399 \\
\hline N704 & $\begin{array}{l}\text { M. Ridell, N118; M. Magnusson, 45; ATCC } 6864 \text {; NCTC } \\
\text { 1935; rabbit in Sumatra }\end{array}$ \\
\hline N705 & M. Riddell, N119; M. Magnusson, 844; nocardiosis, Sweden \\
\hline N707, N708 & M. Ridell, N125, N126; M. Magnusson, 878, 952 \\
\hline N900, N901 & $\begin{array}{l}\text { J. L. Stanford, School of Pathology, Middlesex Hospital, } \\
\text { London, UK, N36, N37; generalized nocardiosis }\end{array}$ \\
\hline N903 & K. P. Schaal, N7 \\
\hline $\mathrm{N} 909 \dagger$ & K. P. Schaal, N66; ATCC 3318 \\
\hline N911 & K. P. Schaal, N199; R. E. Gordon, 3419 \\
\hline N912 & K. P. Schaal, N228; V. A. Orchard, 255 ; soil \\
\hline N963 & $\begin{array}{l}\text { R. Testa, American Cyanamid Co., Lederle Laboratories, Pearl } \\
\text { River, NY, USA, KL1192 }\end{array}$ \\
\hline NS56 & S. M. El-Sanousi, KF9/14, bovine lymph node, Sudan \\
\hline NS67, NS68 & S. M. El-Sanousi, O/80, $0 / 47$, cow milk, Sudan \\
\hline NA488 & M. Abdulle, mammary gland, bovine farcy, Somalia \\
\hline $\begin{array}{l}\text { N. otitidis-caviarum } \\
{ }^{*} \mathrm{~N} 36\end{array}$ & NCTC 1934; E. P. Snijders: infected ear of guinea-pig \\
\hline
\end{tabular}


Table 1. (cont.)

\begin{tabular}{|c|c|}
\hline $\begin{array}{l}\text { Designation and } \\
\text { laboratory number }\end{array}$ & Source and strain history \\
\hline \multicolumn{2}{|l|}{ N. pinensis } \\
\hline N1117 & $\begin{array}{l}\text { L. L. Blackall, NM102; sewage treatment plant, Burleigh Park, } \\
\text { Queensland, Australia }\end{array}$ \\
\hline \multicolumn{2}{|l|}{ N. seriolae } \\
\hline *N1116 & $\begin{array}{l}\text { JCM } 3360 ; \text { K. Hatai, NA8191; spleen of a yellow tail (Seriola } \\
\text { quinqueradiata), Nagasaki, Japan }\end{array}$ \\
\hline N1115 & $\begin{array}{l}\text { JCM } 3359 ; \mathbf{K} \text {. Hatai, kidney of a yellow tail ( } S \text {. } \\
\text { quinqueradiata), Nagasaki, Japan }\end{array}$ \\
\hline N1117 & $\begin{array}{l}\text { JCM } 3361 ; \mathrm{K} \text {. Hatai; S-1; epidermis of a yellow tail ( } S \text {. } \\
\text { quinqueradiata), Kochi, Japan }\end{array}$ \\
\hline N1118 & $\begin{array}{l}\text { JCM } 5849 ; \text { K. Hatai, NA8231; spleen of a yellow tail }(S . \\
\text { quinqueradiata), Nagasaki, Japan }\end{array}$ \\
\hline \multicolumn{2}{|r|}{ 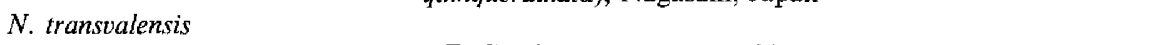 } \\
\hline *N680 & R. E. Gordon, 3426; NCTC 2392 \\
\hline \multicolumn{2}{|l|}{ N. vaccinii } \\
\hline *N682 & $\begin{array}{l}\text { R. E. Gordon; ATCC 11092; N. R. Smith, BG19; stem galls } \\
\text { on blueberry }\end{array}$ \\
\hline \multicolumn{2}{|l|}{$\begin{array}{l}\text { Genus Rhodococcus: } \\
\text { R. aichiensis }\end{array}$} \\
\hline *N934, N938 & M. Tsukamura, E9028, E7776; sputum \\
\hline \multicolumn{2}{|l|}{ R. chlorophenolicus } \\
\hline${ }^{*} \mathrm{~N} 1053$ & $\begin{array}{l}\text { J. H. A. Apajalahti, Department of General Microbiology, } \\
\text { University of Helsinki, Finland, PCP 1; lake sediment }\end{array}$ \\
\hline N1131 & $\begin{array}{l}\text { M. Häggblom, Institute of Environmental Medicine, New } \\
\text { York University Medical Center, NY, USA, CP-2; } \\
\text { contaminated soil }\end{array}$ \\
\hline $\mathrm{N} 1132$ & $\begin{array}{l}\text { M. Häggblom, CG-1; sludge from a wastewater treatment } \\
\text { plant }\end{array}$ \\
\hline \multicolumn{2}{|r|}{ 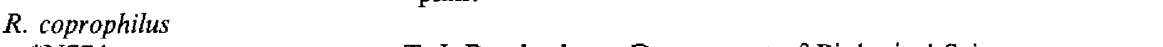 } \\
\hline$* N 774$ & $\begin{array}{l}\text { T. J. Rowbotham, Department of Biological Sciences, } \\
\text { University of Bradford, UK, CUB } 687 \text {; lake mud, } \\
\text { Hawksworth Mere, Guiseley, Leeds, UK }\end{array}$ \\
\hline \multicolumn{2}{|r|}{ the } \\
\hline${ }^{*} \mathrm{C} 7$ & $\begin{array}{l}\text { D. Jones, Department of Microbiology, University of } \\
\text { Leicester, UK, NCTC } 1621 \text {; R. S. Robinson; H. Magnusson; } \\
\text { foal, lung abscess }\end{array}$ \\
\hline R39 & ATCC 25694 \\
\hline \multicolumn{2}{|l|}{ R. erythropolis } \\
\hline *N11 & NCIB 9158; M. Kocur, BS 277; P. H. H. Gray, O-5 \\
\hline N41 & NCIB $8863 ;$ M. E. Brown; chalk soil \\
\hline \multicolumn{2}{|l|}{ R. fascians } \\
\hline${ }^{*} \mathrm{~N} 1062$ & $\begin{array}{l}\text { NCPPB } 3067 ; \text { ATCC 12974; W. H. Burkholder CF17; P. E. } \\
\text { Tilford }\end{array}$ \\
\hline \multicolumn{2}{|l|}{ R. globerulus } \\
\hline${ }^{*} \mathrm{R} 58$ & ATCC 25714 ; R. E. Gordon, 544 \\
\hline $\mathrm{R} 43$ & ATCC $25710 ;$ R. F. Gordon, 494 \\
\hline \multicolumn{2}{|l|}{$\begin{array}{l}\text { 'R. (Corynebacterium) } \\
\text { hoagii' }\end{array}$} \\
\hline$* \mathrm{C} 24$ & hoagit \\
\hline \multicolumn{2}{|r|}{ 19 } \\
\hline *N1008 & $\begin{array}{l}\text { O. A. Nesterenko, Institute of Microbiology and Virology, } \\
\text { Kiev, Ukraine, IMV } 385 \text { soil }\end{array}$ \\
\hline \multicolumn{2}{|r|}{ (1) } \\
\hline${ }^{*} \mathrm{~N} 1056$ & NCIMB 2246; H. Weyland, 3438W; marine sediment \\
\hline \multicolumn{2}{|r|}{ (2) } \\
\hline${ }^{*} \mathrm{~N} 1009$ & O. A. Nesterenko, IMV 195; soil \\
\hline \multicolumn{2}{|r|}{ 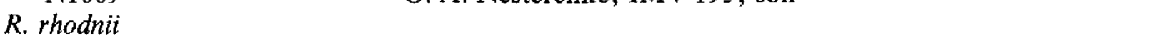 } \\
\hline$* \mathrm{~N} 445$ & $\begin{array}{l}\text { P. Hill, Department of Zoology, University of Edinburgh, UK, } \\
\text { B/0; intestinal tract of Rhodnius prolixus }\end{array}$ \\
\hline \multicolumn{2}{|r|}{ 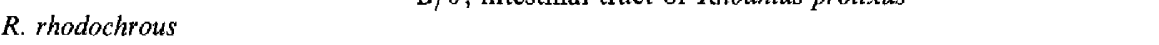 } \\
\hline *N54 & $\begin{array}{l}\text { ATCC 13808; R. E. Gordon, 372; R. S. Breed KMRh; Kral } \\
\text { collection; W. Migula; W. Zopf }\end{array}$ \\
\hline $\begin{array}{l}\text { N65 } \\
\text { R. ruber }\end{array}$ & R. E. Gordon, A7698; H. W. Schoening \\
\hline *N361 & M. Tsukamura, M-1; I. Uesaka; N. M. McClung \\
\hline
\end{tabular}


Table 1. (cont.)

\begin{tabular}{ll}
\hline \multicolumn{1}{c}{$\begin{array}{c}\text { Designation and } \\
\text { laboratory number }\end{array}$} & \multicolumn{1}{c}{ Source and strain history } \\
\hline $\begin{array}{l}\text { Genus Tsukamurella: } \\
\text { T. paurometabola } \\
{ }^{*} \text { N663 } \\
\text { M333 }\end{array}$ & NCTC 10741; M. Tsukamura, 3462; A. Kruse, sputum \\
M334 & R. E. Gordon, IMRU 1283; G. Altmann, 4479; human eye \\
M338 & R. E. Gordon, IMRU 1520; C. McDurnont, R456 \\
M340 & R. E. Gordon, IMRU 586; A. J. Kluyver; N. L. Söhngen \\
& R. E. Gordon, IMRU 340; L. K. Georg, 44-45; iceballs, Hong \\
M347 & Kong \\
& R. E. Gordon; E. L. Biberstein, 106-11B; pneumonic lung of \\
T. wratislaviensis & snail \\
${ }^{*}$ N802 & R. E. Gordon, IMRU 669; N. M. McClung, 33; soil \\
N800 & R. E. Gordon, IMRU 469; R. L. Starkey, 213; soil \\
N801 & R. E. Gordon, IMRU 563; N. M. McClung, 2; soil \\
N803, N805 & R. E. Gordon, IMRU 876, IMRU 878; D. M. Powelson, J-11, \\
N806, N808 & J-17 \\
N809 & R. E. Gordon, IMRU 1385, IMRU 1396; J. J. Perry, R 22, 35 \\
\hline \hline
\end{tabular}

* Type strain. † Duplicated culture. † Strains received as killed, freeze-dried biomass.

Names in inverted commas are not in the Approved Lists of Bacterial Names (Skerman et al., [980).

Abbreviations: ATCC, American Type Culture Collection, Rockville, MD, USA; CBS, Centraalbureau voor Schimmelcultures, Baarn, The Netherlands; DSM, German Collection of Micro-organisms, D-3300 Braunschweig, Germany; JCM, Japan Collection of Micro-organisms, RIKEN, Saitama, Japan; NCDO, National Collection of Dairy Organisms, Shinfield, Reading, UK; NCIB and NCIMB, National Collection of Industrial and Marine Bacteria, Aberdeen, UK; NCPPB, National Collection of Plant Pathogenic Bacteria; Harpenden, UK; NCTC, National Collection of Type Culture, Central Public Health Laboratories, London, UK.

position in the chain (Goodfellow et al., 1978; Daffé et al., 1988).

Mycobacterial mycolic acids are precipitated from ether solutions by ethanol whereas mycolates from corynebacteria, nocardiae and rhodococci remain in solution or, in some cases, give a sparse precipitate (Kanetsuna \& Bartoli, 1972; Hecht \& Causey, 1976; ElSanousi \& Tag El-Din, 1986); the behaviour of mycolates from gordonae and tsukamurellae has not been examined in such systems. The aim of the present study was to design an improved mycolic acid precipitation technique to distinguish mycobacteria from all other mycolic-acidcontaining actinomycetes.

\section{Methods}

Maintenance of test strains. Most of the test strains (Table 1) were maintained on glucose yeast extract agar medium (GYEA; Gordon \& Mihm, 1962) at room temperature and as $20 \%(\mathrm{v} / \mathrm{v})$ glycerol suspensions at $-20^{\circ} \mathrm{C}$ (Wellington \& Williams, 1978). The gordonae, rhodococci and tsukamurellae were maintained on GYEA medium supplemented with thiamin $\left(50 \mathrm{mg} \mathrm{l}^{-1}\right)$, and the corynebacteria on nutrient agar containing Tween $80(0 \cdot 3 \%, \mathrm{v} / \mathrm{v})$.

Cultivation of the test strains. Most of the Mycobacterium strains (Table 1) were grown on GYEA plates for $7-14 \mathrm{~d}$ at $37^{\circ} \mathrm{C}$, checked for purity and then used to inoculate 1 litre flasks containing $250 \mathrm{ml}$ modified Saunton's broth (Mordarska et al., 1972). Inoculated flasks were shaken on an orbital shaker (Gallenkamp) at 150 r.p.m. for up to $28 \mathrm{~d}$ at $37^{\circ} \mathrm{C}$. After checking for purity, the organisms were killed with formalin $(1 \%, v / v)$, harvested by centrifugation, washed twice with distilled water, freeze-dried and kept in universal bottles at room temperature. Some mycobacterial strains (Table 1) were obtained as killed freeze-dried biomass. The gordonae, rhodococci and tsukamurellae were grown in shake flasks of modified Sauton's broth at $30{ }^{\circ} \mathrm{C}$ for $2-5 \mathrm{~d}$, and the corynebacteria in shake flasks of nutrient broth (Oxoid) supplemented with Tween $80(0 \cdot 3 \%, v / v)$ for $3-5 \mathrm{~d}$ as described by Collins et al. (1982).

Extraction of mycolic acids. Dried biomass from the 252 representatives of the genera Corynebacterium, Gordona, Mycobacterium, Nocardia, Rhodococcus and Tsukamurella (Table I) was degraded using alkaline methanolysis to obtain crude mycolic acid methyl esters (MAME; Minnikin, 1988). Biomass (50 mg) in an $8.5 \mathrm{ml}$ Corning tube fitted with a polytetrafluoromethane-lined cap was heated with $2 \mathrm{ml}$ $5 \%(\mathrm{w} / \mathrm{v})$ aqueous tetrabutylammonium hydroxide at $100^{\circ} \mathrm{C}$ overnight. After cooling, the mixture was centrifuged, the supernatant transferred to another Corning tube containing dichloromethane $(2 \mathrm{ml})$ and iodomethane $(25 \mu \mathrm{l})$, shaken for $30 \mathrm{~min}$, centrifuged, and the upper layer discarded. The lower layer, which contained the MAME, was evaporated to dryness under nitrogen at $37^{\circ} \mathrm{C}$.

Precipitation of mycolic acids. The crude dried MAME were dissolved in dichloromethane $(0.5 \mathrm{ml})$, transferred to weighed, $1.5 \mathrm{ml}$ polypropylene microcentrifuge tubes (Treff, Dagersheim, Switzerland) then evaporated to dryness under nitrogen. The residues were shaken with 
toluene $(0.2 \mathrm{ml})$ and acetonitrile $(0.1 \mathrm{ml})$ until completely dissolved, when a further $0.2 \mathrm{ml}$ acetonitrile was added to each preparation. Turbidity upon addition of acetonitrile was indicative of the insolubility of the MAME. The contents of the tubes were mixed very gently, and the preparations kept at $4^{\circ} \mathrm{C}$ for $1 \mathrm{~h}$, then centifuged at 2000 r.p.m. for 2-3 min and the supernatants transferred to another tube. Both precipitates and supernatants were dried under a gentle flow of nitrogen, weighed, and kept at $4{ }^{\circ} \mathrm{C}$ for further analysis. The procedure was repeated with organisms that gave sparse precipitates.

Thin-layer chromatography of mycolic acids. Analytical onedimensional TLC was performed using pieces $(10 \times 10 \mathrm{~cm})$ of aluminium-backed plates (Merck 5554 silica gel $60 \mathrm{~F}_{254}$ ). Dried MAME were dissolved in $100 \mu \mathrm{l}$ light petroleum (b.p. $60-80^{\circ} \mathrm{C}$ ); $2 \mu \mathrm{l}$ solution was applied to the plates, which were then developed twice in light petroleum (b.p. $\left.60-80^{\circ} \mathrm{C}\right) /$ diethyl ether $(85: 15$, v/v; Minnikin et al., 1975). Separated components were revealed by spraying with $5 \%$ ethanolic molybdophosphoric acid followed by heating at $180^{\circ} \mathrm{C}$ for $15 \mathrm{~min}$.

Pyrolysis gas chromatography. Mycolates from selected strains (Table 2) were examined by pyrolysis gas chromatography using a modification of the procedure described by Lechevalier $e t$ al. (1971). The representative dried mycolates were dissolved in $100 \mu \mathrm{l}$ light petroleum (b.p. $60-80^{\circ} \mathrm{C}$ ) and $5 \mu \mathrm{l}$ aliquots injected into the system inlet of a Perkin-Elmer F11 instrument fitted with a column $(3 \mathrm{~m} \times 3.2 \mathrm{~mm})$ packed with $3 \%$ OV- 17 , on Chromosorb WHP. The oven temperature was $270^{\circ} \mathrm{C}$, the injection port temperature was $300^{\circ} \mathrm{C}$ and nitrogen was the carrier gas. Components of the chromatograms were identified by comparing their retention times with those of authentic standards.

\section{Results}

\section{Precipitation of mycolic acid methyl esters}

The 146 representatives of the 28 Mycobacterium species gave copious white precipitates of MAME $[0.5-4 \mathrm{mg}$ (50 mg dry biomass) $\left.{ }^{-1}\right]$ when solutions in acetonitrile/ toluene $(1: 2 \mathrm{v} / \mathrm{v})$ were treated with further acetonitrile (Fig. 1; Table 2). Abundant white precipitates were also consistently recovered from the type strains of Nocardia seriolae (N1116) and Rhodococcus chlorophenolicus (N1053), and less dense ones from Gordona sputi N931, Nocardia farcinica $\mathrm{N} 699$ and the remaining strains of Rhodococcus chlorophenolicus (N1131, N1132). In contrast, The MAME of the 32 representatives of the genera Corynebacterium and Tsukamurella remained in solution, as did those of the remaining strains of Gordona and Rhodococcus.

Thin layer chromatography of mycolic acid methyl esters

All the test strains were found to contain MAME with characteristic mobilities on TLC of the crude methyl mycolate preparations; representative results are shown in Figure 2. Multispot mycolate patterns were obtained with all mycobacteria and for the Tsukamurella paurometabola strains. The latter gave two spots with $R_{F}$ values similar to those of mycobacteria. In contrast, the

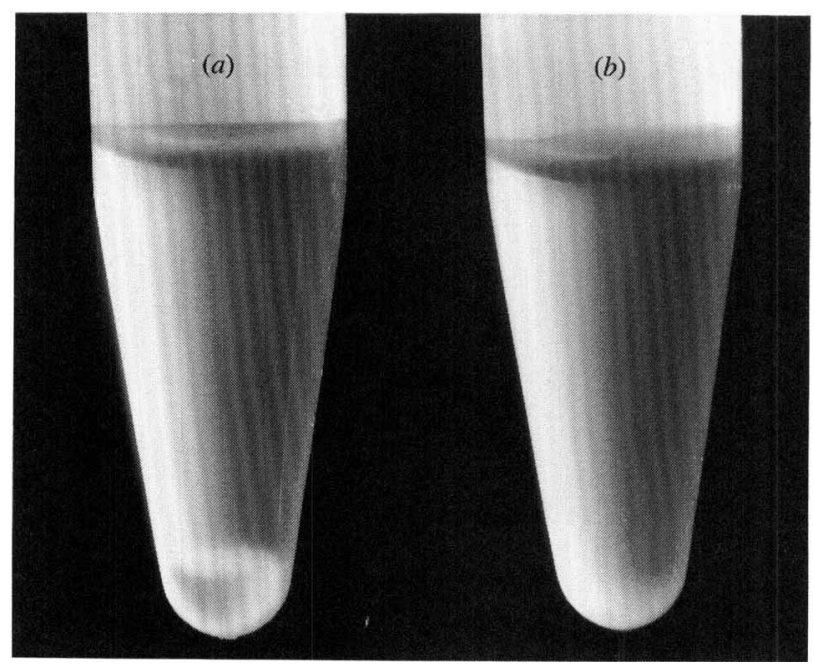

Fig. 1. Precipitation of mycolic acid methyl esters in acetonitrile $(0.3 \mathrm{ml})$ and toluene $(0.2 \mathrm{ml}) .\left(\right.$ a) Mycobacterium farcinogenes ${ }^{*} \mathrm{M} 262$ (note white precipitate in tube) and (b) Nocardia asteroides ${ }^{*} \mathrm{~N} 317$ (absence of precipitate). ${ }^{*}$ Type strains.

Table 2. Dry weight of mycolic acid precipitates derived from representative mycobacteria and some related actinomycetes after addition of a mixture of acetonitrile and toluene $(3: 2, v / v)$ to the crude mycolates, together with details of the major fatty acid esters released on

pyrolysis gas chromatography of the precipitated mycolates

\begin{tabular}{|c|c|c|}
\hline Organisms & 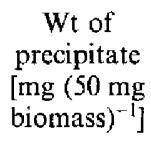 & $\begin{array}{l}\text { Major esters } \\
\text { released on } \\
\text { pyrolysis gas } \\
\text { chromatography }\end{array}$ \\
\hline $\begin{array}{c}\text { Mycobacterium } \\
\text { M504 }\end{array}$ & $4 \cdot 0$ & $\mathrm{C}_{24}$ \\
\hline $\begin{array}{c}\text { 'M. borstelense' } \\
\text { M491 } \\
\text { M487 } \\
\text { M488 }\end{array}$ & $\begin{array}{l}1 \cdot 5 \\
1 \cdot 5 \\
2 \cdot 0\end{array}$ & $\begin{array}{l}\mathrm{C}_{24} \\
\mathrm{C}_{24} \\
\mathrm{C}_{24}\end{array}$ \\
\hline $\begin{array}{c}\text { M. bovis BCG } \\
\text { M461 }\end{array}$ & $3 \cdot 5$ & $\mathrm{C}_{26}$ \\
\hline $\begin{array}{c}\text { M. chelonei } \\
\text { *M350 } \\
\text { M438 } \\
\text { M442 } \\
\text { M441 }\end{array}$ & $\begin{array}{l}0 \cdot 5 \\
1 \cdot 5 \\
1 \cdot 0 \\
1 \cdot 0\end{array}$ & $\begin{array}{l}\mathrm{C}_{24} \\
\mathrm{C}_{24} \\
\mathrm{C}_{24} \\
\mathrm{C}_{24}\end{array}$ \\
\hline $\begin{array}{l}\text { M. chitae } \\
{ }^{*} \mathrm{M} 101\end{array}$ & $2 \cdot 5$ & $\mathrm{C}_{24}$ \\
\hline $\begin{array}{c}\text { M. farcinogenes } \\
\text { *M262 } \\
\text { M270 } \\
\text { M272 } \\
\text { M277 } \\
\text { M284 } \\
\text { M466 } \\
\text { M468 } \\
\text { M473 }\end{array}$ & $\begin{array}{l}4 \cdot 0 \\
3 \cdot 0 \\
4 \cdot 0 \\
2 \cdot 5 \\
3 \cdot 5 \\
2 \cdot 0 \\
2 \cdot 5 \\
3 \cdot 0\end{array}$ & $\begin{array}{l}\mathrm{C}_{24} \\
\mathrm{C}_{24} \\
\mathrm{C}_{24} \\
\mathrm{C}_{24} \\
\mathrm{C}_{24} \\
\mathrm{C}_{24} \\
\mathrm{C}_{24} \\
\mathrm{C}_{24}\end{array}$ \\
\hline
\end{tabular}


Table 2. (cont.)

\begin{tabular}{|c|c|c|}
\hline Organisms & $\begin{array}{c}\text { Wt of } \\
\text { precipitate } \\
{[\mathrm{mg}(50 \mathrm{mg}} \\
\left.\text { biomass })^{-1}\right]\end{array}$ & $\begin{array}{c}\text { Major esters } \\
\text { released on } \\
\text { pyrolysis gas } \\
\text { chromatography }\end{array}$ \\
\hline M474 & $2 \cdot 5$ & $\mathrm{C}_{24}$ \\
\hline M476 & $4 \cdot 0$ & $\mathrm{C}_{24}$ \\
\hline M481 & $2 \cdot 0$ & $\mathrm{C}_{24}^{4}$ \\
\hline M482 & $2 \cdot 5$ & $\mathrm{C}_{24}^{24}$ \\
\hline N710 & $3 \cdot 0$ & $\mathrm{C}_{24}$ \\
\hline N711 & $2 \cdot 0$ & $\mathrm{C}_{24}^{4}$ \\
\hline N720 & 1.5 & $\mathrm{C}_{24}^{2}$ \\
\hline \multicolumn{3}{|l|}{ M. fortuitum } \\
\hline$*_{\mathrm{M} 204}$ & $4 \cdot 0$ & $\mathrm{C}_{24}$ \\
\hline M205 & $3 \cdot 0$ & $\mathrm{C}_{24}$ \\
\hline M368 & $2 \cdot 0$ & $C_{24}^{24}$ \\
\hline M369 & 3.0 & $\mathrm{C}_{24}^{4}$ \\
\hline M527 & $2 \cdot 0$ & $\mathrm{C}_{24}$ \\
\hline \multicolumn{3}{|l|}{ M. peregrinum } \\
\hline *M6 & 0.5 & $\mathrm{C}_{24}$ \\
\hline M418 & 0.5 & $\mathrm{C}_{24}$ \\
\hline M419 & $1 \cdot 0$ & $\mathrm{C}_{24}$ \\
\hline M421 & 0.5 & $\mathrm{C}_{24}^{44}$ \\
\hline M422 & 0.5 & $\mathrm{C}_{24}$ \\
\hline \multicolumn{3}{|l|}{ M. senegalense } \\
\hline${ }^{*} \mathrm{M} 263$ & $2 \cdot 5$ & $\mathrm{C}_{24}$ \\
\hline M264 & $1 \cdot 5$ & $\mathrm{C}_{24}^{4}$ \\
\hline M268 & $2 \cdot 0$ & $\mathrm{C}_{24}^{24}$ \\
\hline N718 & $2 \cdot 5$ & $\mathrm{C}_{24}$ \\
\hline N721 & $2 \cdot 5$ & $\mathrm{C}_{24}$ \\
\hline $\mathrm{N} 723$ & $2 \cdot 0$ & $\mathrm{C}_{24}$ \\
\hline \\
\hline${ }^{*} \mathrm{M} 216$ & $3 \cdot 0$ & $\mathrm{C}_{24}$ \\
\hline \multicolumn{3}{|l|}{ M. tuberculosis. } \\
\hline \\
\hline $\begin{array}{l}\text { Gordona sput } \\
\text { N931 }\end{array}$ & $>0.5$ & $\mathrm{C}_{16}-\mathrm{C}_{18}$ \\
\hline Nocardia farcinica & $>0.5$ & $\mathrm{C}_{12}-\mathrm{C}_{18}$ \\
\hline $\begin{array}{l}\text { Nocardia seriolae } \\
{ }^{*} \text { N11116 }\end{array}$ & $2 \cdot 5$ & $\mathrm{C}_{12}-\mathrm{C}_{18}$ \\
\hline \multicolumn{3}{|l|}{$\begin{array}{l}\text { Rhodococcus } \\
\text { chlorophenolicus }\end{array}$} \\
\hline
\end{tabular}

* Type strain.

Tsukamurella wratislaviensis strains gave single mycolic acid spots with $R_{F}$ values comparable to the single spots produced by Gordona $\left(R_{F} 0.35\right)$ and Nocardia $\left(R_{F} 0.35\right)$ strains. The corynebacteria and rhodococci also produced single spots, with slightly lower $R_{F}$ values $(0 \cdot 26-0 \cdot 29)$.

\section{Pyrolysis gas chromatography}

The major methyl ester obtained from methyl mycolates from most mycobacteria was $\mathrm{C}_{24}$ (Table 2). However, $M$.

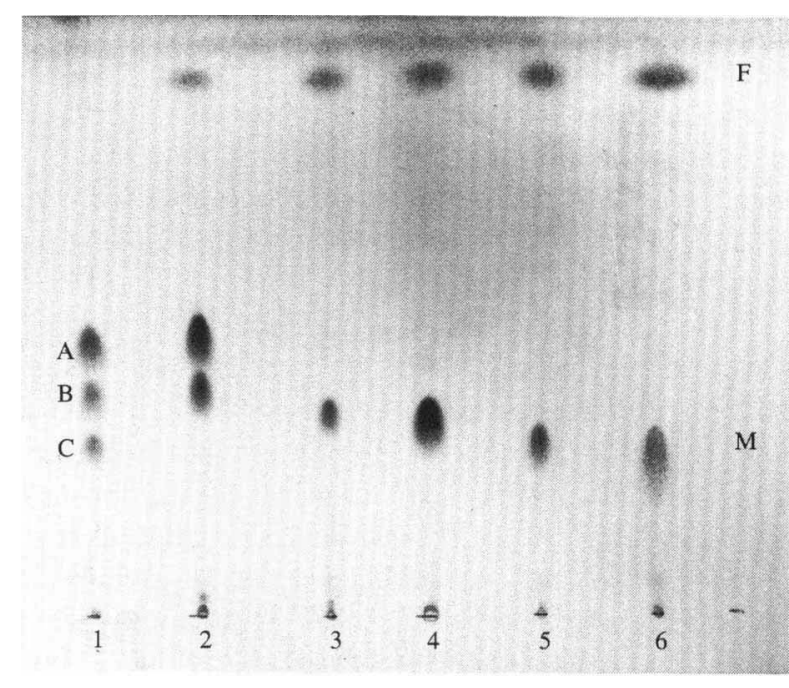

Fig. 2. One-dimensional TLC of methyl mycolates of Mycobacterium tuberculosis C (lane 1); Tsukamurella paurometabola M334 (lane 2); Gordona bronchialis N1058 (lane 3); Nocardia asteroides *N317 (lane 4); Rhodococcus rhodochrous *N54 (lane 5); Corynebacterium diphtheriae PW8 (lane 6). Developing solvent: light petroleum (b.p. $\left.60-80^{\circ} \mathrm{C}\right) /$ diethyl ether $(85: 15, \mathrm{v} / \mathrm{v}, \times 2)$. Detection agent, $5 \%$ ethanolic molybdophosphoric acid followed by charring at $180^{\circ} \mathrm{C}$. Abbreviations: M, MAME; A, $\alpha$-mycolates; $B$, methoxymycolates: $C$, ketomycolates; $\mathrm{F}$, non-hydroxylated fatty acid methyl esters (note the absence of this spot in lane 1 because fatty acid esters were not precipitated). ${ }^{*}$ Type strains.

bovis and $M$. tuberculosis strains released $\mathrm{C}_{26}$ as the predominant methyl ester. Methyl esters corresponding to $\mathrm{C}_{20}, \mathrm{C}_{22}$ and $\mathrm{C}_{26}$ components were found in relatively small amounts in several of the mycobacterial strains. Rhodococcus chlorophenolicus N1053 gave a pattern of methyl esters characteristic of mycobacteria. In contrast, MAME from G. sputi N931, N. farcinica $\mathrm{N} 699$ and $N$. seriolae $\mathrm{N} 1116$, like other representatives of the genera Gordona and Nocardia, released esters in the range of $\mathrm{C}_{12}-\mathrm{C}_{18}$.

\section{Discussion}

The mycolic acid precipitation method described above is more reliable and sensitive than existing precipitation procedures designed to separate mycobacteria from the other mycolic-acid-containing taxa (Kanetsuna \& Bartoli, 1972; Hecht \& Causey, 1976; El-Sanousi \& Tag ElDin, 1986). Hecht \& Causey (1976), for example, noted that many corynebacteria, nocardiae and rhodococci gave weak precipitates of MAME. The present method also requires smaller amounts of biomass than the procedures described by Kanetsuna \& Bartoli (1972) and El-Sanousi \& Tag El-Din (1986). The basis of the method is the solubility of all MAME in acetonitrile/ 
toluene $(1: 2, \mathrm{v} / \mathrm{v})$ and the insolubility of those from mycobacteria in acetonitrile/toluene $(3: 2, \mathrm{v} / \mathrm{v})$. These solvent mixtures were found to be superior to the diethyl ether and methanol or ethanol mixtures used previously. There is an advantage in having two relatively high boiling solvents so that no evaporation takes place during the procedure.

The selective insolubility of mycobacterial mycolates in acetonitrile/toluene $(3: 2, \mathrm{v} / \mathrm{v})$ can be attributed to structural differences between these mycolic acids and those from other mycolic-acid-containing actinomycetes. Mycobacterial mycolic acids have 60-90 carbon atoms with 1-3 double bonds; the corresponding figures for the remaining taxa are: corynebacteria, $22-38$ carbons with $0-2$ double bonds; gordonae, $48-66$ carbons with 1-3 double bonds; nocardiae, 44-60 carbons with 0-3 double bonds; rhodococci, 34-52 carbons with 0-2 double bonds; and tsukamurellae, 64-78 carbons with 1-6 double bonds (Minnikin \& Goodfellow, 1980; Goodfellow, 1991; Hartmans \& De Bont, 1991). The lower ratio of double bonds to mycolic acid chain length in non-mycobacterial mycolates helps to explain the lower melting points and increased solubility of these components in polar solvents such as acetonitrile.

Mycobacterial mycolic acids also include a range of other functional groups such as cyclopropane rings, methyl branches, keto, methoxy, ester or epoxy groups but only cis-double bonds are found in the other taxa. The positioning of the double bonds and other functional groups within the molecule may be an important factor governing the physical properties of these acids. In mycobacteria, the groups are almost equally spaced down the main chain but in members of the other taxa the double bonds are concentrated towards the terminal methyl end of the chain. More data are needed to determine precisely the reasons for the different physical behaviour between mycobacterial mycolates and those from the other taxa but the observed difference provides a practical way of distinguishing the genus $\mathrm{Myco}$ bacterium.

Pyrolysis gas chromatography may be used to confirm the presence of mycobacterial methyl mycolates in the precipitates as these substances characteristically release $\mathrm{C}_{20}-\mathrm{C}_{26}$ fatty acid esters on pyrolysis (Minnikin \& Goodfellow, 1980; Hartmans \& De Bont, 1991). The pyrolysis products derived from non-mycobacterial methyl mycolates fall within the following ranges: $\mathrm{C}_{8}-\mathrm{C}_{18}$ for corynebacteria, $\mathrm{C}_{16}-\mathrm{C}_{18}$ for gordonae, $\mathrm{C}_{20}-\mathrm{C}_{22}$ for tsukamurellae, and $\mathrm{C}_{12}-\mathrm{C}_{18}$ for nocardiae and rhodococci. The range of pyrolytic products obtained from the mycobacterial and non-mycobacterial methyl mycolates extracted in the present study are in line with the results from previous reports (Lechevalier et al., 1971; Goodfellow et al., 1978; Collins et al., 1982; Luquin et al., 1991). This simple form of pyrolysis gas chromatography requires only a routine chromatograph with a temperature-controlled inlet system without a special pyrolyser.

It would appear from the precipitation and pyrolysis gas chromatography data that the type strain of $R$. chlorophenolicus contains mycolic acids typical of mycobacteria. This is unexpected since in the original description of this taxon the type strain was reported to have mycolic acids with 33-43 carbons atoms (Apajalahti et al., 1986). Further comparative taxonomic studies are needed to determine the taxonomic status of $R$. chlorophenolicus (or whether cross-contamination has occurred). In contrast, our pyrolysis gas chromatographic data support the current classification of the other nonmycobacterial strains which gave a positive result in the precipitation test, namely $G$. sputi N931, $N$. seriolae N1116 and N. farcinica N699.

It is clear from the present investigation that the mycolic acid precipitation method provides a means of distinguishing mycobacteria from other mycolic acidcontaining taxa complementing corresponding TLC techniques (Minnikin et al., 1975, 1980, 1984a, $b, 1985$ ). It was notable that the $M$. fallax and $M$. triviale strains gave dense white precipitates of methyl mycolates; these organisms are unusual amongst mycobacteria in giving single mycolic acid spots on TLC analysis of wholeorganism methanolysates (Dobson et al., 1985) so that they may be identified as non-mycobacterial. Conversely, as confirmed in the present study, $T$. paurometabola strains are unusual in producing multispot mycolate patterns on TLC that can be confused with those formed by some mycobacteria (Goodfellow et al., 1978; Collins et al., 1988). However, the MAME of Tsukamurella remain in solution in the precipitation test. Tsukamurella paurometabola and $T$. wratislaviensis strains can be readily distinguished from each other as the methyl mycolates of the latter form a single spot in TLC analyses.

It can be concluded from the present investigation that the mycolic acid precipitation test provides a reliable and practical means of distinguishing mycobacteria from all other bacteria. The test should be particularly useful in distinguishing mycobacteria from members of other mycolic-acid-containing taxa when more sophisticated methods are unavailable. Improved diagnostic tests are, for instance, needed to distinguish Nocardia farcinica from Mycobacterium farcinogenes and Mycobacterium senegalense strains. The latter are the principal agents of bovine farcy, a chronic infectious disease of zebu cattle that occurs in some parts of the tropics. This disease was originally considered to be caused by $N$. farcinica, but mycolic acid analysis helped to show that the aetiological agent was a Mycobacterium (Asselineau et al., 1969). In 
the present study all 64 representatives of $M$.farcinogenes and $M$. senegalense gave dense white precipitates of methyl mycolates whereas the corresponding mycolates of 21 of the $22 \mathrm{~N}$. farcinica strains remained in solution upon addition of acetonitrile and toluene. The exception, $N$. farcinica $\mathrm{N} 699$, gave a weak positive result but was clearly shown to be correctly classified as the precipitated mycolates produced fatty acid esters typical of nocardiae on pyrolysis gas chromatography.

This research was supported by a studentship from the Sudanese Government (M.E.H.). The authors are also grateful to Mr R. C. Bolton for technical assistance and to colleagues (see Table 1) who kindly provided strains.

\section{References}

Apajalahti, J. H. A., Kärpänoja, P. \& Salkinoja-Salonen, M. S. (1986). Rhodococcus chlorophenolicus sp. nov., a chlorophenolmineralising actinomycete. International Journal of Systematic Bacteriology 36, 246-251.

Asselineau, J., Lanéelle, M. A. \& Chamolseau, G. (1969). De l'etiologie du farcin du zébus tchadiens: nocardiose ou mycobactériose? II. Composition lipidique. Revue d' Élévage et de Médicine Vétérinaire des Pays Tropicaux 22, 205-209.

BOWDEN, G. H. \& GoOdFEllow, M. (1990). The actinomycetes: Actinomyces, Nocardia and related genera. In Topley and Wilson's Principles of Bacteriology, Virology and Immunology, 8th edn, vol. 2, Systematic Bacteriology, pp. 31-57. Edited by M. T. Parker \& B. I. Duerden. London: Edward Arnold.

Collins, M. D., Goodfellow, M. \& Minnikin, D. E. (1982). A survey of the structure of mycolic acids in Corynebacterium and related taxa. Journal of General Microbiology 128, 129-149.

Collins, M. D., Smida, J., Dorsch, M. \& Stackebrandr, E. (1988) Tsukamurella gen. nov. harboring Corynebacterium paurometabolum and Rhodococcus aurantiacus. International Journal of Systematic Bacteriology 38, 385-391.

Daffé, M., LanÉElle, M. A. \& Valero Guillen, P. L. (1988) Tetraenoic and pentaenoic mycolic acids from Mycobacterium thamnopheos, structure, taxonomic and biosynthetic implications. European Journal of Biochemistry 177, 339-344.

Dobson, G., Minnikin, D. E., Minnikin, S. M., Parlett, J. H., Goodfellow, M., Ridell, M. \& Magnusson, M. (1985). Systematic analysis of complex mycobacterial lipids. In Chemical Methods in Bacterial Systematics, pp. 237-265. Edited by M. Goodfellow \& D. E. Minnikin. London: Academic Press.

EL-SANousi, S. M. \& TAG EL-Din, M. H. (1986). On the aetiology of bovine farcy in the Sudan. Journal of General Microbiology 132, $1673-1675$

Goodfellow, M. (1991). The family Nocardiaceae. In The Prokaryotes. A Handbook on the Biology of Bacteria: Ecophysiology, Isolation, Identification, Applications, 2nd edn, pp. 1188-1213. Edited by A. Balows, H. G. Trüper, M. Dworkin, W. Harder \& K. H. Schleifer. New York: Springer-Verlag.

Goodfellow, M., Orlean, P. A. B., Collins, M. D., Alshamaony, L. \& MinNikin, D. E. (1978). Chemical and numerical taxonomy of strains received as Gordona aurantiaca. Journal of General Microbiology 109, 57-68.
Gordon, R. E. \& Mihm, J. M. (1962). Identification of Nocardia caviae (Erikson) nov. comb. Annals of the New York Academy of Sciences 98, 628-636.

Hartmans, S. \& De Bont, J. (1991). The genus Mycobacteriumnonmedical. In The Prokaryotes. A Handbook on the Biology of Bacteria: Ecophysiology, Isolation, Identification, Applications, 2nd edn, pp. 1214-1237. Edited by A. Balows, H. G. Trüper, M. Dworkin, W. Harder \& K. H. Schleifer. New York: Springer-Verlag.

Hecht, S. T. \& CAUSEY, W. A. (1976). Rapid method for the detection and identification of mycolic acids in aerobic actinomycetes and related bacteria. Journal of Clinical Microbiology 4, 284-287.

KanetsUNA, F. \& BaRTOLI, A. (1972). A simple chemical method to differentiate Mycobacterium from Nocardia. Journal of General Microbiology 70, 209-212.

Lechevaliek, M. P., Horan, A. C. \& LecheValier, H. A. (1971). Lipid composition in the classification of nocardiae and mycobacteria. Journal of Bacteriology 105, 313-318.

Luquin, M., Ausina, V., Lopez-Calahorra, F., Belda, F., GarciaBarcelo, M., Celma, C. \& Prats, G. (1991). Evaluation of practical chromatographic procedures for identification of clinical isolates of mycobacteria. Journal of Clinical Microbiology 29, 120-130.

MinNikIN, D. E. (1988). Isolation and purification of mycobacterial wall lipids. In Bacterial Cell Surface Techniques, pp. 125-135. Edited by I. C. Hancock \& I. R. Poxton. Chichester: J. Wiley.

Minnikin, D. E. \& Goodfellow, M. (1980). Lipid composition in the classification and identification of acid-fast bacteria. In Microbiological Classification and Identification, pp. 189-256. Edited by M. Goodfellow \& R. G. Board. London: Academic Press.

Minnikin, D. E., Alshamaony, L. \& Goodfellow, M. (1975). Differentiation of Mycobacterium, Nocardia and related taxa by thinlayer chromatography analysis of whole organism methanolysates. Journal of General Microbiology 88, 200-204.

Minnikin, D. E., Hutchinson, I. G., Caldicott, A. B. \& GoodFELLOW, M. (1980). Thin-layer chromatography of methanolysates of mycolic acid-containing bacteria. Journal of Chromatography $\mathbf{1 8 8}$, 221-233.

Minnikin, D. E., Minnikin, S. M., Hutchinson, I. G., Goodfellow, M. \& GRANGE, J. M. (1984a). Mycolic acid patterns of representative strains of Mycobacterium fortuitum, 'Mycobacterium peregrinum' and Mycobacterium smegmatis. Journal of General Microbiology 130, 363-367.

Minnikin, D. E., Minnikin, S. M., Parlett, J. H., Goodfellow, M. \& MAGNUSSON, M. (1984b). Mycolic acid patterns of some species of Mycobacterium. Archives of Microbiology 139, 225-231.

Minnikin, D. E., Minnikin, S. M., Parlett, J. H. \& Goodfellow, M. (1985). Mycolic acid patterns of some rapidly-growing species of mycobacteria. Zentralblatt für Bakteriologie und Hygiene A259, 446-460.

MordarsKa, H., Mordarski, M. \& Goodfellow, M. (1972). Chemotaxonomic characters and classification of some nocardioform bacteria. Journal of General Microbiology 71, 77-86.

Skerman, V. B. D., MCGowan, V. \& SNeAth, P. H. A. (1980). Approved lists of bacterial names. International Journal of Systematic Bacteriology 30, 225-420.

Stackebrandt, E., Smida, J. \& Collins, M. D. (1988). Evidence of phylogenetic heterogeneity within the genus Rhodococcus: renewal of the genus Gordona (Tsukamura). Journal of General and Applied Microbiology 34, 341-348.

Wellington, E. M. H. \& Williams, S. T. (1978). Preservation of actinomycete inoculum in frozen glycerol. Microbios Letters 6, 151-157. 